\title{
Desenvolvimento do aplicativo IFG-Estudos para auxiliar a interação entre docentes, discentes e grupos de estudo do IFG-Câmpus Luziânia
}

\author{
Cauã F. Barros ${ }^{1}$, Lara Ma Veloso Andrade², Renan R. de Oliveira ${ }^{3}$, Luiz F. Batista \\ $\operatorname{Loja}^{4}$
}

${ }^{1}$ Instituto Federal de Educação Ciência e Tecnologia de Goiás (IFG) - Campus Luziânia, GO - Brazil

${ }^{2}$ Instituto Federal de Educação Ciência e Tecnologia de Goiás (IFG) - Campus Luziânia, GO - Brazil

${ }^{3}$ Diretoria de Administração Acadêmica

Instituto Federal de Educação Ciência e Tecnologia de Goiás (IFG) - Campus Goiânia, GO - Brazil

${ }^{4}$ Departamento de Áreas Acadêmicas

Instituto Federal de Educação Ciência e Tecnologia de Goiás (IFG) - Campus Anápolis, $\mathrm{GO}$ - Brazil

\{cauafbarros, laramaria.velosoandrade\} @gmail.com, \{renan.rodrigues, luiz. loja\} @ifg.edu.br

\begin{abstract}
Considering the academic experiences, the life experience of the students and making some use of literature, it was observed the need of having, in the educational environment, a tool of easy and practical handling for the promotion of study groups. This finding resulted in the development of a mobile application called IFG-Estudos. This application was initially created for teachers and students of the Instituto Federal de Educação, Ciência e Tecnologia de Goiás - Câmpus Luziânia. This article describes, briefly and through a succinct bibliographical review, the motivations by which the work was developed and the steps taken to achieve the main objective: the development of the IFG-Estudos software.
\end{abstract}

Resumo. Considerando as experiências acadêmicas, a experiência de vida dos alunos e fazendo uso da literatura, observou-se a necessidade de se ter, no meio educacional, uma ferramenta de fácil e prático manuseio para a promoção de grupos de estudo. Essa constatação resultou no desenvolvimento de um aplicativo mobile denominado IFG-Estudos. Esse aplicativo foi criado inicialmente para os docentes e discentes do Instituto Federal de Educação, Ciência e Tecnologia de Goiás - Câmpus Luziânia. Este artigo descreve, ainda que de maneira breve e por meio uma sucinta revisão bibliográfica, as motivações pelas quais o trabalho foi desenvolvido e as etapas percorridas para alcançar o objetivo principal: o desenvolvimento do software IFGEstudos. 
VII Congresso Brasileiro de Informática na Educação (CBIE 2018)

Anais dos Workshops do VII Congresso Brasileiro de Informática na Educação (WCBIE 2018)

\section{Introdução}

Atualmente a busca pelo conhecimento de forma rápida e prática tem se tornado uma necessidade para docentes e discentes. A internet possui um papel importante nessa busca, pois por meio dela essa procura pelo saber é facilitada. Além disso, sabe-se que diversos instrumentos tecnológicos têm sido inseridos no ambiente educacional. Esses instrumentos solucionam vários problemas enfrentados tanto por alunos quanto por professores, potencializando o processo de ensino-aprendizagem.

Uma das dificuldades dos discentes é identificar grupos de estudo relacionados a um assunto específico. Essa dificuldade pode ser causada pela distância entre o aluno e o grupo de estudo, ou o estudante pode não ter conhecimento da existência do grupo por pertencer a uma escola ou turma diferente.

O objetivo principal deste trabalho foi desenvolver um aplicativo para dispositivos móveis que auxilie na localização dos grupos de estudo, facilitando a interação entre os docentes e discentes, beneficiando a troca de experiências e aprendizado. Inicialmente, o aplicativo está sendo implementado para auxiliar os estudantes e professores do Instituto Federal de Educação, Ciência e Tecnologia de Goiás - Câmpus Luziânia, viabilizando a comunicação e facilitando o encontro entre essas pessoas de forma simples e rápida.

\section{Das motivações}

\subsection{Do grupo de estudo}

Como evidencia a Lei de Diretrizes e Bases da Educação Nacional são fins da educação o aprimoramento do educando como pessoa humana, o suscito do desejo permanente de aperfeiçoamento cultural e profissional, o desenvolvimento do pensamento reflexivo e sua preparação para continuar aprendendo (Lei n ${ }^{\circ}$ 9.394, de 20 de dezembro de 1996). A partir dessas diretrizes, os educadores devem se preocupar com a formação de indivíduos autônomos e críticos. Uma das estratégias utilizadas pelos docentes para atingir esse objetivo é a criação de grupos de estudos.

De maneira geral, um grupo de estudo consiste em um conjunto de pessoas que se associam em torno de uma área de interesse comum e se articulam promovendo encontros regulares. A organização de grupos de estudos é uma ideia poderosa e simples, que pode propiciar uma alternativa no apoio ao desenvolvimento profissional (GIMENES e PENTEADO, 2008).

É interessante lembrar que a interação social exerce importante papel na aprendizagem e no desenvolvimento humano. Segundo a abordagem feita por Vigotski, observa-se que, ao conviver com outras pessoas, o indivíduo efetua troca de informações e assim vai construindo o seu conhecimento (MELLO; TEIXEIRA, 2012).

Os grupos de estudo podem auxiliar os alunos a interagirem e aprimorarem seus saberes sobre assuntos de seus interesses. Além disso, os estudantes podem compartilhar ideias com outras pessoas desenvolvendo o pensamento e aprofundando o entendimento (GERDY, 1998 apud WIERSEMA, 2002). Nesse sentido, esse tipo de estratégia se mostra bastante oportuna.

Finalmente, essa estratégia de estudo estreita o relacionamento entre estudantes, fazendo-os entenderem a importância do trabalho em equipe, bem como valorizar suas individualidades para benefício de todos. Assim, em um grupo de estudo a cooperação 
VII Congresso Brasileiro de Informática na Educação (CBIE 2018)

Anais dos Workshops do VII Congresso Brasileiro de Informática na Educação (WCBIE 2018)

entre os participantes é estimulada, o compartilhamento de experiências incentivado e trabalhada a compreensão e resolução de conflitos de forma colaborativa.

\subsection{Dos problemas encontrados}

Apesar dos vários benefícios supracitados, encontrar um grupo de estudo relacionado a um assunto específico pode não ser uma tarefa fácil. Muitos estudantes não participam de grupos de estudo porque não conseguem identificar grupos relacionados aos assuntos de seu interesse. Outro obstáculo enfrentado pelos discentes no momento de procurar um grupo é a timidez (CRAWFORD; TAYLOR 2000). Isto significa que, por serem inibidos, muitos estudantes não conseguem se apresentar em um grupo de pessoas. Dessa forma, essa característica do estudante pode limitá-lo, fazendo com que ele não ingresse em uma equipe.

Além disso, existem estudantes que desconhecem a existência de estratégias colaborativas de estudos e tampouco conhecem o conceito de grupo de estudo. Essa falta de informação os impossibilita utilizar esse recurso de aprimoramento de conhecimentos.

\subsection{Da proposta de solução}

Fundamentados na validade do uso de grupos de estudo para o aprendizado e cientes dos problemas elencados, voltamos nossos olhares para o Instituto Federal de Goiás Câmpus Luziânia - o câmpus responsável pelo desenvolvimento desta pesquisa, o que trouxe melhor conhecimento de causa. Além disso, a vivência da equipe de desenvolvimento no câmpus criou a convicção em entender que esse campus é passivo dos mesmos problemas ditos anteriormente.

Foi a partir disso que a ideia da criação de um aplicativo mobile começou a ganhar espaço. O avanço e popularização dos dispositivos móveis têm proporcionado significativas mudanças nos métodos e nas formas de ensino, aprendizado e estudo tradicionais. Tal avanço se deu, sobretudo, por meio do surgimento de condições favoráveis como as Tecnologias de Informação e Comunicação (TIC's) para o desenvolvimento de abordagens de ensino que incluam aplicações destes dispositivos no ambiente educacional.

É sobre o conceito Mobile Learning que este trabalho é embasado. Podendo ser traduzido para português como "aprendizagem móvel" ou entendido como integração das tecnologias móveis no contexto educativo. O Mobile Learning é uma modalidade de ensino e aprendizagem relativamente recente, que permite a criação de novos ambientes de aprendizagem à distância, utilizando para isso, dispositivos móveis com acesso à Internet.

Dessa forma, o objetivo deste projeto foi desenvolver um aplicativo para dispositivos móveis que auxilie a interação entre docentes, discentes e grupos de estudo no IFG - Câmpus Luziânia. Essa ferramenta possibilitará a criação de grupos de estudo, tanto pelos docentes quanto pelos discentes.

Além disso, o sistema disponibilizará informações sobre quais são os grupos de estudos desenvolvidos no câmpus, como encontrá-los, as áreas de estudo referentes a cada um deles e os respectivos horários de encontros. Essas informações auxiliarão os estudantes a encontrarem e se integrarem a um grupo de estudo fazendo com ele se torne mais autônomo ajudando-o na continuidade da busca por conhecimento.

Assim, a ferramenta proposta neste trabalho funcionará como um facilitador, auxiliando os estudantes a encontrarem uma forma diferenciada de aprendizado. Espera- 
VII Congresso Brasileiro de Informática na Educação (CBIE 2018)

Anais dos Workshops do VII Congresso Brasileiro de Informática na Educação (WCBIE 2018)

se com a utilização da ferramenta que o senso de responsabilidade do estudante se desenvolva à medida que o discente se esforce na busca pelo conhecimento.

\section{Metodologia}

Inicialmente foi realizado um estudo sobre os variados conceitos que envolvem o desenvolvimento do software, nos utilizando de diferentes livros além do apoio dos professores para sanar dúvidas e esclarecer questões. Dando continuidade, estudamos sobre as técnicas de desenvolvimento de software para Internet, aplicações para dispositivos móveis e ferramentas de desenvolvimento.

\subsection{Das ferramentas}

O front-end da aplicação, isto é, a parte do software que interage diretamente com o usuário promovendo a ele uma interface amigável, foi realizado com o auxílio do framework Bootstrap - uma ferramenta gratuita para desenvolvimento HTML, CSS e JavaScript. Amplamente conhecida, é bastante utilizado para criação de sites responsivos e mobile. A linguagem de programação PHP e o sistema gerenciador de banco de dados (SGBD) MySQL ficaram reservados para a implementação da regra de negócio da aplicação, o chamado back-end da aplicação.

Após a finalização do desenvolvimento da aplicação Web adaptável aos dispositivos móveis, foi utilizada o Cordova PhoneGap (framework open source) que permitiu transformar a aplicação web em um aplicativo para dispositivo móvel híbrido.

A preferência na escolha por uma natureza híbrida se deve ao fato de que aplicações nativas são desenvolvidas tendo em vista a execução em uma plataforma específica, o que demanda maior complexidade ao projeto e que por consequência requer maior conhecimento da parte do programador (PIRAN e LAZZARETTI, 2016, p. 3).

Em contrapartida, aplicações híbridas mesclam características tanto das aplicações web, como por exemplo sua menor complexidade no desenvolvimento, quanto das nativas. Uma aplicação híbrida é multiplataforma como a Web, isto é, pode ser executada em várias plataformas diferentes sem alterações significativas no código fonte (PREZOTTO; BONIATI 2014) e ainda tem acesso aos recursos de hardware tal como uma aplicação nativa (FROZZA, s.d).

Nesse contexto, o Cordova PhoneGap se encaixa perfeitamente, sendo um dos frameworks mais utilizados para a criação desse tipo de aplicação. Trata-se de um desenvolvimento mais rápido e de menor custo, fazendo uso de linguagens como HTML5, CSS3, JavaScript, entre outros. Neste contexto, o Cordova PhoneGap é utilizado para empacotar o sistema baseado em tecnologias, transformando-o em uma aplicação mobile, com possibilidade de realização de chamadas de código para chamadas nativas no dispositivo quando necessário. O Cordova PhoneGap cria uma WebView para as diversas plataformas do projeto. O resultado é um app instalável, publicável na loja (e com todas as vantagens que correspondem), porém implementada com um código multiplataforma.

As razões que envolveram a escolha de cada uma das ferramentas do back-end da aplicação foram as seguintes:

1.PHP: linguagem de script open source de uso geral, muito utilizada e

especialmente adequada para o desenvolvimento web (NIEDERAUER, 2005).

2. MySQL: SGBD que possui código aberto e é desenvolvido em PHP. 
VII Congresso Brasileiro de Informática na Educação (CBIE 2018)

Anais dos Workshops do VII Congresso Brasileiro de Informática na Educação (WCBIE 2018)

3. Cordova PhoneGap: framework de código aberto que gera aplicações que acessam às funções nativas dos dispositivos móveis e possibilita a criação de aplicativos móveis híbridos (PIRAN E LAZZARETTI, 2016, p. 7). Atualmente oferece suporte a sete plataformas, dentre elas Android, IOS e Windows Phone.

\subsection{Das etapas do desenvolvimento}

É comum que o desenvolvimento de um software seja uma tarefa melindrosa. Uma alternativa para lidar com sua complexidade compreende o chamado processo de desenvolvimento de software. Um processo de desenvolvimento de software classifica em atividades as tarefas realizadas durante a construção do software (BEZERRA, s.d, p. 19).

Há distintos processos de desenvolvimento, entretanto, segundo Bezerra (s.d, p. 20), existem algumas atividades típicas que são comuns à maioria dos sistemas. São elas:

1.Levantamento de requisitos: etapa de levantamento e definição das necessidades dos futuros usuários do sistema.

2.Análise de requisitos: fase na qual o produto da atividade anterior é estudado e a partir disso, modelos de representação do sistema são construídos.

3.Projeto: fase que determina como o sistema funcionará a fim de atender aos requisitos já levantados, de acordo com os recursos tecnológicos existentes.

4.Implementação: fase na qual o software é codificado, isto é, fase na qual ocorre a tradução da descrição da fase de projeto em código executável.

5.Testes: como o próprio nome sugere, o produto é testado e possíveis erros são reparados.

6.Implantação: o sistema é instalado no ambiente do usuário.

Esse projeto teve por base as referidas etapas. Para construção das representações da etapa de análise de requisitos, foi utilizada a linguagem visual de modelagem de sistemas UML.

O Modelo de Ciclo de Vida do software escolhido foi o iterativo e incremental por sua abordagem mais realista. Nele, o desenvolvimento é dividido em ciclos e em cada um desses ciclos, as fases de análise, projeto, implementação e testes podem ser identificadas.

De acordo com Bezerra (s.d, p. 34), no modelo iterativo e incremental, um sistema é desenvolvido em vários passos similares (iterativo) e em cada passo, o sistema é estendido com mais funcionalidades (incremental). Essa característica permite uma melhor evolução do projeto como um todo, haja vista que todas as etapas estão em constante revisão e adaptação. O mesmo vale também para a escolha das ferramentas. Observou-se a necessidade de mudança das ferramentas estabelecidas anteriormente para o projeto.

O estilo da arquitetura de software escolhido foi o cliente-servidor. Nessa arquitetura o servidor é responsável por oferecer serviços e os clientes podem requisitálos (TANENBAUM, 2009).

\section{Resultados}

É notório que grupos de estudo são importantes aliados no processo de ensinoaprendizagem. Além dos benefícios em termos de conhecimento, o estudante tem a 
VII Congresso Brasileiro de Informática na Educação (CBIE 2018)

Anais dos Workshops do VII Congresso Brasileiro de Informática na Educação (WCBIE 2018)

oportunidade de aprimorar aspectos como relacionamento interpessoal, altruísmo e compreensão da importância do trabalho em equipe.

Sabe-se que desenvolver aplicações híbridas tem sido uma prática recorrente e amplamente utilizada na atual contemporaneidade. Dado o seu baixo custo de desenvolvimento e a sua natureza multiplataforma, esse tipo de aplicação conquistou espaço na elaboração de aplicativos móveis e tem adquirido muitos adeptos.

A união do conceito de grupo de estudo junto à popularização dos dispositivos móveis e smartphones mostrou-se proveitoso, uma vez que permite que a aprendizagem ocorra a qualquer momento em qualquer lugar.
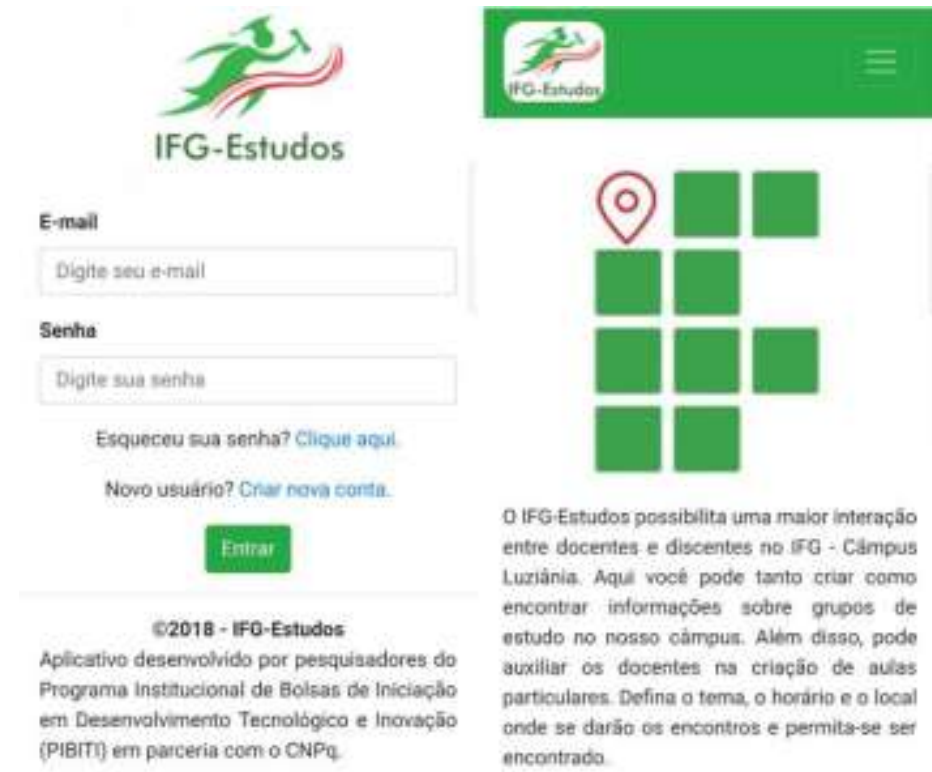

O IFG Estudos possiblita uma maior interaçāo entre docentes e discentes no $15 \mathrm{G}$ - Campus Luziánia Aqua vocé pode tanto criar como encontrar informaçóes sobre grupos de estudo no nosso oàmpun. Alem dissa, pode auxiliar os docentes na criação de aulas particulares Defina o tema o horirio e o local onde se darăo ot encontros e permita-se set encontrado

Figura 1: tela de login, tela inicial e menu de opções

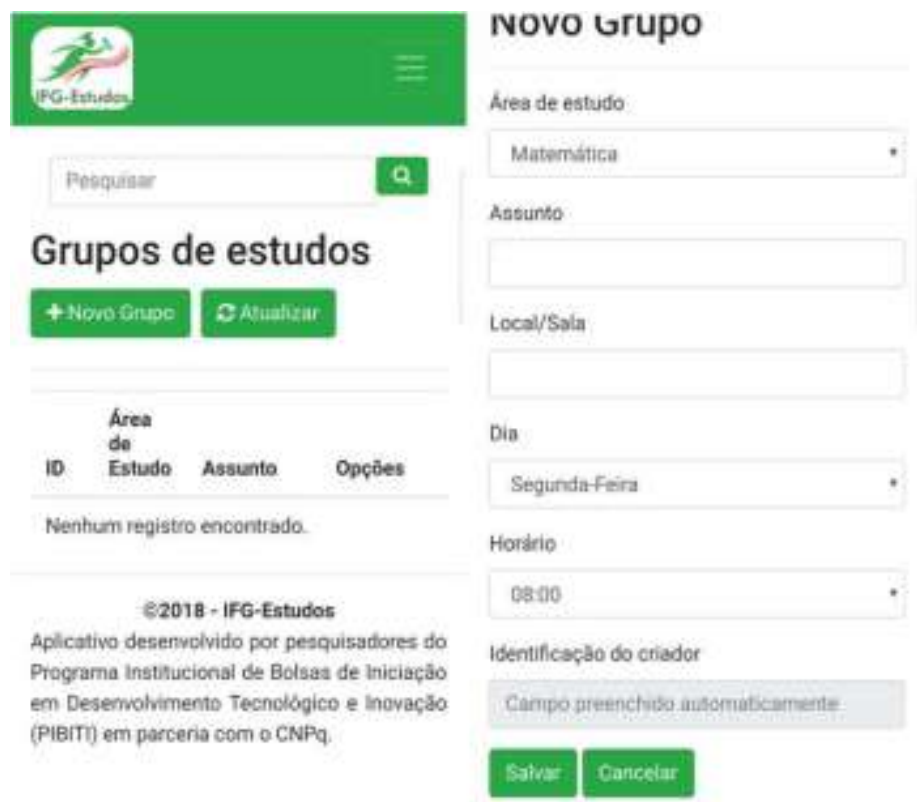

Figura 2: tela de listar grupos e criar grupo 
VII Congresso Brasileiro de Informática na Educação (CBIE 2018)

Anais dos Workshops do VII Congresso Brasileiro de Informática na Educação (WCBIE 2018)

\section{Considerações finais}

Ao longo do trabalho buscou-se elencar as motivações que deram origem ao aplicativo mobile IFG-Estudos, objetivo fim do projeto do Programa Institucional de Bolsas de Iniciação em Desenvolvimento Tecnológico e Inovação (PIBITI) em questão. Além disso, as etapas do desenvolvimento foram citadas a fim de evidenciar o caminho percorrido para a construção do software.

As dificuldades enfrentadas no decorrer do trabalho, contribuíram significativamente para nossa formação enquanto estudantes. A experiência de observar nossa comunidade, identificar problemas e propor soluções foi de grande valia também para nosso crescimento como cidadãos ativos.

O IFG-Estudos teve êxito em sua versão inicial. Como a aplicação não tem fins lucrativos, todos os códigos-fonte serão disponibilizados ao Instituto Federal de Goiás, como de praxe.

Pretende-se dar continuidade ao projeto reparando funcionalidades já existentes e adicionando novas funcionalidades com o propósito de melhorá-lo, incluindo, a melhoria da interface com usuário proporcionando uma experiência fluída com aparência semelhante a outros aplicativos do mesmo ambiente. Visa-se também a publicação da aplicação nas lojas oficiais das principais plataformas para que o acesso seja facilitado.

\section{Referências}

BEZERRA, Eduardo. Princípios de análise e projeto de sistemas com UML. $3^{\text {a }}$ Tiragem.

Elsevier: Editora Campus, s.d.

BOOTSTRAP. About Bootstrap. Disponível em: <https://getbootstrap.com.br/docs/4.1/getting-started/introduction/>. Acesso em: 09 ago. 2017.

BRASIL. Lei n. 9.394, de 20 de dezembro de 1996. Estabelece as diretrizes e bases da educação nacional. Disponível em: <http://www.planalto.gov.br/ccivil_03/leis/L9394.htm>. Acesso em: 12 abr. 2017.

CRAWFORD, Lynne; TAYLOR, Linda. Timidez, esclarecendo suas dúvidas. São Paulo: Agora, 2000. 120 p.

ESPÍNDOLA, Rafaela. Como funciona o Mobile Learning? Edools, 2016. Disponível em: <https://www.edools.com/mobile-learning/>. Acesso em: 14 ago. 2018.

GIMENES, J.; PENTEADO, M. G. Aprender Matemática em grupo de estudos: uma experiência com professoras de séries iniciais. ZETETIKÉ - Cempem - FE Unicamp - v. $16-$ n. $29-$ jan./jun. - 2008. Disponível em: <http://www.portal.santos.sp.gov.br/seduc/e107_files/downloads/formacoes/gefoco_a bril_-_2010.pdf>. Acesso em 13 abr. 2017.

MELLO, Elisângela de Fátima Fernandes de; TEIXEIRA, Adriano Canabarro. A interação social descrita por Vigotski e a sua possível ligação com a aprendizagem colaborativa através das tecnologias de rede. In: ANPED SUL, IX., 2012, Caxias do Sul - $\quad$ RS. $\quad$ Disponível em: < http://www.ucs.br/etc/conferencias/index.php/anpedsul/9anpedsul/paper/viewFile/6/8 7>. Acesso em: 30 set. 2018. 
VII Congresso Brasileiro de Informática na Educação (CBIE 2018)

Anais dos Workshops do VII Congresso Brasileiro de Informática na Educação (WCBIE 2018)

NIEDERAUER, Juliano. Desenvolvendo Website com PHP. $2^{\circ}$ Edição. São Paulo: Novatec Editora, 2005.

PHONEGAP. About The Project. Disponível em: <http://phonegap.com/about/>. Acesso em: 13 abr. 2017.

PHPMYADMIN. About PHPMyAdmin. Disponível em: <https://www.phpmyadmin.net/>. Acesso em: 09 ago. 2017.

PIRAN, Férlon. M.; LAZZARETTI, Alexandre. T. Estudo da tecnologia PhoneGap/Cordova e a aplicação em um estudo de caso. 2016. Disponível em: <http://painel.passofundo.ifsul.edu.br/uploads/arq/20160331164543877842707.pdf>. Acesso em: 10 abr. 2017.

PREZOTTO, E. D.; BONIATI, B. B. Estudo de Frameworks Multiplataforma Para Desenvolvimento de Aplicações Mobile Híbridas. 2014. Disponível em: <http://www.eati.info/eati/2014/assets/anais/artigo8.pdf>. Acesso em: 15 abr. 2017.

TANENBAUM, Andrew S. Sistemas operacionais modernos. $3^{\circ}$ Ed. São Paulo: Pearson Prentice Hall, 2009.

WIERSEMA, Nico. How does Collaborative Learning actually work in a (Mexican) classroom and how do students react to it? A Brief Reflection. 2002. Disponível em: <https://eric.ed.gov/?id=ED464510>. Acesso em: 13 abr. 2017. 